\title{
P 108 'TAKING THE CLINIC TO THE PATIENT'
}

Joy Waldock. Mid Yorkshire Hospitals NHS Trust, Wakefield, England

10.1136/bmjspcare-2014-000654.149

Background Palliative care patients often struggle to attend outpatient clinics as they reach the end of life, preferring to be seen at home by Macmillan nurses. Sometimes a medical review is needed but as consultants often work across different settings with fixed commitments, 'ad hoc' home visits can be difficult.

Hospitals gain income from outpatient attendances and there is a pressure to ensure clinics are full and non attendance rates are kept low.

Within MYH (population 500,000) there were 4 palliative medicine outpatient clinics each week in three different hospitals. An average of 14\% of clinic slots were filled. In October 2012 the decision was made to 'take the clinic to the patient' and one consultant changed the clinic and to offer 'slots' for home visits. Aims The aim of this evaluation was to see how many patients were seen at home in a 6 month period, whether they would 
have been well enough to attend a clinic and to assess the symptoms seen.

Results 1-3 patients/session

94\% had cancer

$41 \%$ had pain, $43 \%$ other symptoms, 16\% psychological issues (some overlap)

$38 \%$ were well enough to attend an outpatient clinic, $41 \%$ were not

In 21\% might have been 'possible but difficult'

Several patients were seen in the last 2 weeks of life, a situation where a clinic attendance is not likely to be appropriate.

Conclusions/recommendations Numbers are small, there is capacity for more visits.

Patients were seen who would not have managed to get to a clinic.

There was still capacity in the remaining 3 clinics.

Arrangements are informal but seem to suit the community team.

Having one fixed day to do visits is helpful from the consultant's organisational point of view but may not be as responsive as ideal for the community team. 